\title{
Intervención didáctica para la extracción y cuantificación de esteroides en orina
}

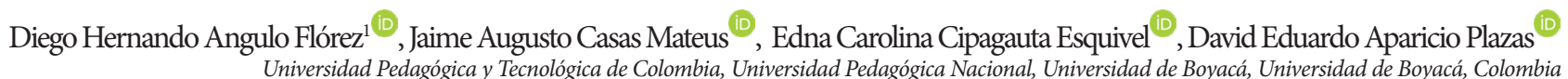

Autor de correspondencia: 1diego.angulo@uptc.edu.co Recibido: 15 de junio de 2020 Revisado: 08 agosto de 2020 Aprobado: 29 de diciembre de 2020 Publicado: 25 de marzo de 2021

\section{Resumen}

Este artículo describe una estrategia didáctica para la enseñanza de un método de extracción en fase sólida miniaturizada en contextos del trabajo práctico de laboratorio [TPL]. Se parte de los imaginarios previos de los estudiantes para, a partir de algunas sesiones de laboratorio, generar cambios conceptuales y promover un aprendizaje significativo. En este trabajo se relatará la experiencia de conjugar el empleo de una técnica de preparación de muestras - PT-SPE - con un ejercicio de intervención didáctica para el desarrollo de habilidades procedimentales. Se presentan los resultados de la aplicación de TPL en la multideterminación de tres esteroides en muestras de orina al emplear HPLC-DAD-PT-C18-SPE, que presentan adecuados porcentajes de recuperación y parámetros de validación. Los resultados de la intervención muestran una mejoría en habilidades relacionadas con métodos de extracción en fase sólida. Se concluye que hubo cambios conceptuales frente a procesos analíticos, una adecuada familiarización con nuevas metodologías de preparación de muestras, aumento significativo del lenguaje químico y la comprensión de conceptos, junto con la aplicación de procesos cognitivos de bajo orden, para la promoción de habilidades cognitivas de alto orden.

Palabras clave: didáctica de las ciencias experimentales, trabajos prácticos de laboratorio, preparación de muestras, SPE, HPLC 


\title{
Didactic intervention for the extraction and quantification of steroids in urine
}

\begin{abstract}
This article describes a didactic strategy for teaching a miniaturized solid-phase extraction method in the context of laboratory practical training [LPT]. It is based on the students' previous imaginaries in order to generate conceptual changes and promote meaningful learning through a few laboratory sessions. This article will report on the experience of combining the use of a sample preparation technique-PT-SPE-with a didactic intervention exercise for the improvement of procedural skills. We present the results of the application of LPT in the multi-determination of three steroids in urine samples using HPLC-DAD-PT-C18-SPE, which show adequate recovery rates and validation parameters. The results of the intervention show improvement of skills related to solid-phase extraction methods. It is concluded that there were conceptual changes in analytical processes, an adequate familiarization with new sample preparation methodologies, a significant increase in chemical vocabulary, and understanding of concepts, along with the application of lower-order cognitive processes to the enhancement of higher-order cognitive skills.
\end{abstract}

Keywords: experimental science didactics, laboratory practical training, sample preparation, SPE, HPLC

\section{Intervenção didática para a extração e quantificação de esteroides na urina}

\section{Resumo}

O artigo descreve uma estratégia didática para o ensino de um método de extração em fase sólida miniaturizado no contexto da prática laboratorial [PL]. Isto se baseia nos imaginários prévios dos estudantes a fim de gerar mudanças conceituais e promover um aprendizado significativo através de algumas sessões de laboratório. Este artigo relatará a experiência de combinar o uso de uma técnica de preparação de amostras - PT-SPE - com um exercício de intervenção didática para o desenvolvimento de habilidades processuais. Apresentam-se os resultados da aplicação de PL na determinação múltipla de três esteroides em amostras de urina usando HPLC-DAD-PT-C18-SPE, mostrando percentuais de recuperação e parâmetros de validação adequados. Os resultados da intervenção mostram uma melhoria das habilidades relacionadas aos métodos de extração em fase sólida. Conclui-se que houve mudanças conceituais nos processos analíticos, uma familiarização adequada com as novas metodologias de preparação de amostras, um aumento significativo no uso do vocabulário químico e na compreensão de conceitos, além da aplicação de processos cognitivos de baixa ordem para a promoção de habilidades cognitivas de ordem mais elevada.

Palavras-chave: didática das ciências experimentais, prática laboratorial, preparação de amostras, SPE, HPLC 
Los procedimientos analíticos requieren técnicas de preparación de muestras como un paso esencial para obtener resultados confiables, lo cual garantiza la reproducibilidad y disminuye la profusión de errores, en pro de una confiabilidad estadística de los datos obtenidos (Alcántara-Durán et al., 2018; Angulo et al., 2018). En esta perspectiva, la microextracción en fase sólida emerge como una alternativa viable, en virtud de la rapidez del análisis, de la reducción de la muestra y de volúmenes de disolventes, de la simplicidad de su empleo, así como de sus posibilidades para su automatización (Liu et al., 2017).

La extracción en fase solida adaptada en puntera PT-SPE - pipette-tip solid phase extraction- es uno de los métodos más prometedores para la preparación de muestras miniaturizadas de extracción en fase sólida (Angulo et al., 2018; Jiang et al., 2018; Liu et al., 2017; Yang et al., 2017). Se diferencia de la SPE - solid phase extraction - convencional por precisar de una cantidad mínima de material adsorbente y no necesitar ningún dispositivo de extracción adicional, excepto una jeringa comercial. En general, es una técnica de bajo costo por la cual la extracción del analito es más rápida y fácil en comparación con el uso del SPE convencional (Amiri \& Ghaemi, 2017; Nacimento et al., 2018; Pelit et al., 2015). La extracción en fase sólida adaptada en puntera - nombre adoptado para las puntas de las pipetas de precisión- puede ser empleada con diversos materiales adsorbentes, entre los que se encuentran los polímeros conductores y molecularmente impresos, nanotubos de carbono, filtro de cigarro - celulosa-y el C18 convencional o mesoporoso (Gañán et al., 2016; Razmkhah et al., 2018).

Por otro lado, la separación cromatográfica ocurre a partir de la distribución y la interacción entre los componentes de una fase móvil y otra estacionaria (Ponzetto et al., 2017). En los equipos de HPLC, la alta presión es compatible con el sistema cromatográfico. Además, la sofisticación del equipo y las nuevas fases estacionarias aumentan la efectividad de las separaciones, lo que reduce el tiempo de análisis y aumenta la versatilidad y la automatización (Chiesa et al., 2016; Li et al., 2015; Nezhadali et al., 2016).

Además de lo anterior, en lo que tiene que ver con el plano didáctico, la transposición didáctica es definida como el 'trabajo' que transforma un objeto de saber en un objeto de enseñanza (Ghibaudi et al., 2016). Desde tal perspectiva, un contenido de saber que ha sido designado como saber a enseñar sufre a partir de entonces una serie de transformaciones adaptativas que lo hacen apto para ser un objeto de enseñanza. El concepto de transposición didáctica se remite al paso del saber sabio — científico - al saber enseñado — didáctico-, y, por lo tanto, a la distancia eventual obligatoria que los separa.

La figura 1 ilustra una serie de transformaciones que van de lo implícito a lo explícito, de la práctica a la teoría, de lo preconstruido a lo construido (Dănescu, 2015), en tal estructura cognitiva primeramente evidenciada en los científicos, pero también en los estudiantes o en la población objetivo. La transposición didáctica comprende una serie de etapas de transformación producidas en el conocimiento desde su elaboración misma por parte de la comunidad científica hasta su destino como conocimiento escolar (Hassan et al., 2015). Por otra parte, el saber científico no puede ser enseñado tal cual como este se encuentra en los textos científicos. Esto es un obstáculo en el proceso de aprendizaje, por lo que debe ser modificado o traducido como un saber a enseñar. Por una parte, el saber científico se presenta en textos técnicos. El saber a enseñar se encuentra casi siempre en libros de 
naturaleza 'didáctica', algunos programas y en materiales de apoyo (Kampelmann et al., 2018).

Figura 1

Proceso de transposición didáctica

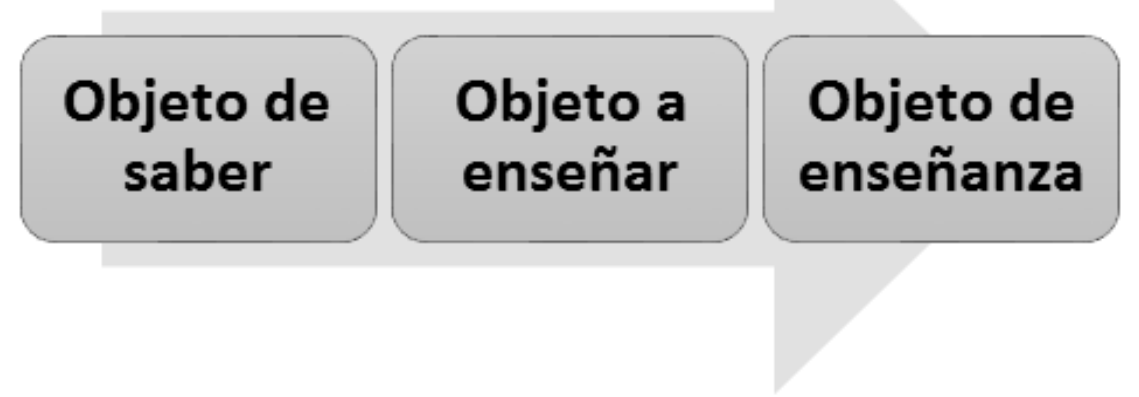

Por su parte, en la enseñanza de las ciencias ha surgido una serie de cuestionamientos sobre la importancia de las prácticas de laboratorio. Desde la óptica de los adeptos, esta investigación recoge la premisa de entender que tanto profesores como estudiantes encuentran diferentes finalidades a las prácticas de laboratorio, que en alguna medida son desvirtuadas si desde el inicio de las prácticas el docente no motiva, ni orienta para que los estudiantes vean el verdadero sentido de estas actividades. No obstante, los estudiantes normalmente disfrutan cuando trabajan en el laboratorio (Chen et al., 2016). Con frecuencia, los estudiantes tienen agrado por las prácticas de laboratorio solo cuando conocen lo que van a realizar; cuando no, pierden el interés con facilidad y se les dificulta su aprendizaje. Para esto, es imperativo hacer un experimento adecuado - con objetivos claros y que funcione $-\mathrm{y}$ tener una medida de control e independencia suficientes (Chen et al., 2016). También se sabe que en algunas experiencias los estudiantes no adquieren ningún tipo de destrezas en el escenario de laboratorio. Desde esta comprensión, la adquisición de técnicas o destrezas del laboratorio pueden tener poco valor en sí mismas, en la medida que no permitan desarrollar habilidades de tipo práctico como manipulación de instrumental preciso - balanzas, balones aforados, pipetas, buretas, entre otros- (Bostan et al., 2015).

Con base en lo anterior, los trabajos prácticos de laboratorio [TPL], como elemento esencial diseñado para que los estudiantes adquieran una mejor idea de la naturaleza de la ciencia y de las investigaciones científicas (Franco et al., 2017), surgen como una herramienta didáctica que permite descubrimiento, lo cual favorece el cambio conceptual (Hernández, 2012). Además, el trabajo experimental es fundamental en la enseñanza de las ciencias. Existen metodologías que favorecen el aprendizaje de conceptos, el desarrollo de habilidades y de la capacidad para resolver problemas (Qing et al., 2010).

Facilitar por completo el procedimiento experimental, el marco teórico, los objetivos y los resultados no genera interés en el estudiante, lo que se refleja en el hecho que, cuando el docente proporciona el marco conceptual, deja poco espacio para la construcción del significado personal, desde una posición que ejerce el control sobre la identificación del problema, la generación de hipótesis, el diseño experimental y los métodos que se emplean 
para manipular e interpretar los datos obtenidos desde la observación (Bostan et al., 2015; Sudhoff et al., 2020). Al estimular el desarrollo y la intensificación conceptuales para animar a los estudiantes a explorar, elaborar y supervisar sus propias ideas —concepciones alternativas, imaginarios o ideas previas-, en comparación con las aportadas desde el experimento científico, se puede afirmar que el trabajo de laboratorio tendrá un papel relevante (Bostan et al., 2015).

Estudios han demostrado que la mayoría de los técnicos de laboratorio y los profesionales de la química desconocen las técnicas miniaturizadas de preparación de muestras. Por lo tanto, su utilización solo es explotada en ambientes universitarios (Hernández, 2012; RojasHernández \& Ramírez-Silva, 2012; Sevian \& Fulmer, 2012). Por lo anterior, en este trabajo se estudiaron las ventajas de enseñar la extracción en fase sólida adaptada en puntera, a partir de la identificación de las ideas previas de los estudiantes, entendidas como aquellas ideas que están en desacuerdo con el conocimiento científicamente aceptado e influyen en el aprendizaje de nuevos conocimientos científicos (Özmen et al., 2009). Es bien sabido que las ideas previas son ampliamente compartidas por los estudiantes en varios niveles o grados de aceptación. Además, los imaginarios previos son lógicos, sensibles y valiosos desde el punto de vista de los estudiantes, así como generalizados, estables y resistentes al cambio mediante las estrategias de enseñanza tradicionales (Dönmez \& Ayas, 2010). Con base en la identificación de las ideas previas y en la aplicación de los TPL, se procedió a implementar la técnica dentro de los trabajos experimentales de análisis químico instrumental, para extrapolar - a futuro, posiblemente- su uso a los ambientes de investigación en ciencias químicas y a la didáctica de las ciencias experimentales.

\section{Método}

\section{Reactivos y solventes}

- Patrones analíticos: Progesterona, PGN 98.55 \% -Tianjin Wanhua Technology Development Co. Ltda ${ }^{\oplus}$, Tianjin, China-, Prednisolona, PRe $98.55 \%$-Genix Pharmaceutical Corp ${ }^{\star}$, Vancouver, Canadá- y Estradiol, EsD 99.03 \% —Olon Ricerca Bioscience ${ }^{\circledast}$, Concord, EE. UU.- .

- Solventes grado HPLC: tolueno, acetona, tetrahidrofurano y cloroformo -J.T. Baker ${ }^{\oplus}$, Ciudad de México, México-.

- Material C18 para SPE y para PT-SPE —Sigma-Aldrich ${ }^{\circledR}$, Steinheim, Alemania-.

- Solventes grado HPLC: metanol, acetonitrilo, isopropanol obtenidos -Dinâmica ${ }^{\oplus}$, Diadema, Brasil-.

- El agua fue purificada en un sistema Milliporo Milli-Q Plus -Bedford, EE. UU-.

\section{Instrumentación y condiciones de separación}

Para el análisis cromatográfico, se empleó un sistema HPLC Agilent 1290 -Agilent Technologies, Palo Alto, EE, UU-, compuesto por una bomba cuaternaria -G1311 B-, inyector automático modelo 1260 Hip ALS - G1367E- y un detector DAD modelo 1290 $\mathrm{VL}+-\mathrm{G} 1315 \mathrm{C}$ - . Todas las separaciones de los analitos fueron realizadas en una columna analítica Phenomenex ${ }^{\circledR}$ Gemini C18 $-150 \mathrm{~mm} \times 4,60 \mathrm{~mm}, 5 \mu \mathrm{m}$, Torrance, EE, UU-. La fase móvil consistió en una mezcla de acetonitrilo: metanol $(45+55, \mathrm{v} / \mathrm{v})$ y fue bombeada 
a un flujo de $0,85 \mathrm{ml} \mathrm{min}^{-1}$. Los datos cromatográficos fueron adquiridos a $265 \mathrm{~nm}$ con detección DAD. El volumen de inyección fue de $40 \mu \mathrm{l}$ para los patrones y las muestras. Se utilizó el programa Agilent Open LAB Cromatography Data System ${ }^{\circledR}$ para controlar el sistema y la adquisición de datos.

\section{Preparación de muestras con el uso de PT-C18-SPE}

Se llenó una puntera de $1 \mathrm{ml}$ con algodón esterilizado en la punta con el material adsorbente -40 mg de C18-. Otra porción de algodón fue empleada para fijar el material. La activación con el dispositivo se realizó con $0,1 \mathrm{ml}$ de agua ultrapura utilizando una jeringa comercial (figura 2). El eluyente se evaporó y el residuo se resuspendió con $150 \mu \mathrm{l}$ de metanol para inyectar $40 \mu \mathrm{l}$ en el HPLC-DAD.

\section{Figura 2}

Dispositivo de extracción en fase sólida adaptado en puntera PT-C18-SPE

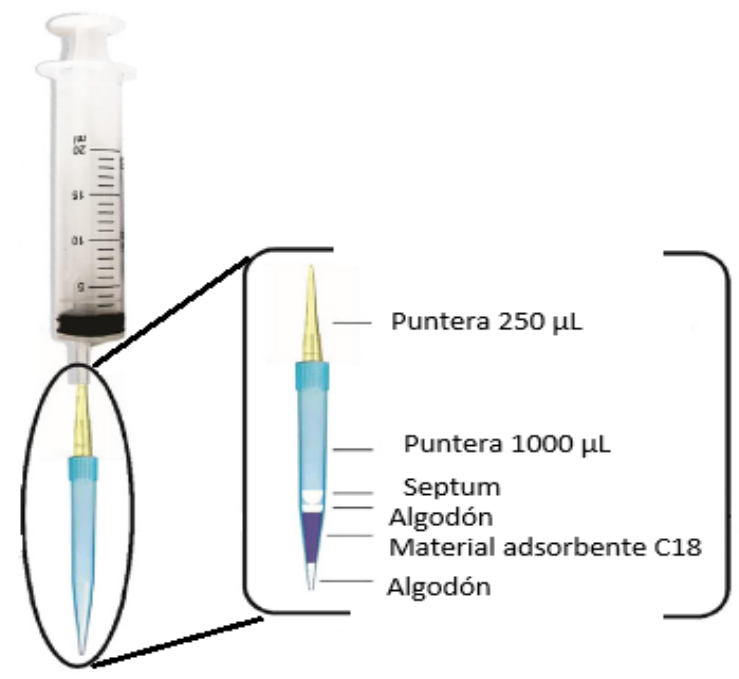

\section{Validación del método}

Para la validación del método se tuvieron en cuenta la sensibilidad, el límite de cuantificación [LOQ], la linealidad, la precisión, la exactitud, la robustez y la estabilidad. Las curvas de calibración analítica se realizaron mediante estándares externos basados en inyecciones de las soluciones preparadas a concentraciones de 16, 40, 100, 200, 500, 800 y $1200 \mathrm{ng} \mathrm{ml}^{-1}$, extraídas por triplicado. El LOQ se evaluó a partir de seis ensayos analíticos de orina enriquecida $(250 \mu \mathrm{l}, \mathrm{n}=6)$ a concentraciones decrecientes para cada analito (tabla 1 ). Los resultados de precisión y exactitud se expresaron en RE \% - error relativo- y RSD \% —desviación estándar relativa-, respectivamente. Los estudios de precisión y exactitud se realizaron seis veces para demostrar la repetitividad del método. La precisión intermedia se realizó en tres días diferentes $(\mathrm{n}=3)$, a concentraciones de 40, 200 y $800 \mathrm{ng} \mathrm{ml}^{-1}$.

La robustez fue analizada a partir de la capacidad del método analítico de permanecer inalterado por pequeñas variaciones en el procedimiento. Se determinó al variar parámetros como flujo ( $\pm 0,10$ unidades), volumen de inyección ( $\pm 0,10$ unidades) y composición de la fase móvil $-40 \%$ de acetonitrilo $+60 \%$ de metanol, $45 \%$ de acetonitrilo $+55 \%$ de metanol, $50 \%$ de 
acetonitrilo $+50 \%$ de metanol- . Las concentraciones de residuos de los analitos se evaluaron con la prueba t de Student, con un nivel de significación establecido en un valor de $\mathrm{p} \leq 0,05$.

\section{Intervención didáctica}

Como el objetivo de esta investigación era quelos estudiantes de química - de bacharelado da Universidade Federal de Sao Joao del Rei, del espacio analítica instrumental- tuvieran una alternativa de preparación de muestras más rápida, económica y versátil, fue necesaria una ardua preparación, teórica y experimental en referencia a las técnicas citadas. Esto se consiguió con sesiones sobre el tema y prácticas de laboratorio para que los estudiantes esclarecieran los conceptos y las destrezas necesarias para realizar la preparación de muestras con la técnica implementada, con el fin de lograr una transposición didáctica, desde un entorno científico a un entorno de aprendizaje. Para conseguir lo antes expuesto, se realizaron los siguientes procedimientos:

Prueba para la determinación de ideas previas. A manera global, se buscaba determinar qué sabían los estudiantes antes de la intervención en el aula. En el instrumento se hizo una breve descripción sobre lo que es la metodología de SPE y las variaciones miniaturizadas como la PT-SPE. El instrumento constó de cinco preguntas. Finalmente, para la evaluación de los resultados que se obtuvieron, se elaboró una matriz de valoración.

Exposición sobre la técnica PT-SPE y PT-C18-SPE. Para esta fase de la intervención didáctica se realizó la descripción de las etapas de esta técnica de preparación de muestras, con énfasis en las posibles ventajas de la técnica sobre la SPE convencional.

Procedimiento experimental para la determinación de esteroides por PT-C18-SPEHPLC. Para esta práctica de laboratorio se diseñó un procedimiento en el que se realizó la determinación de los analitos seleccionados al emplear PT-C18-SPE como método de preparación de muestras, teniendo en cuenta que se quería comparar la efectividad de la técnica en términos de recuperación de analitos, así comola funcionalidad del procedimiento de preparación de muestras en comparación con la técnica de SPE convencional. La práctica se llevó a cabo con siete grupos de cinco personas. Cada grupo realizó el proceso de determinación de los analitos por SPE y PT-SPE y después intercambió los procedimientos con el objetivo de determinar los analitos con el empleo de ambas técnicas. Finalmente, los estudiantes debían entregar un informe en donde se evidenciarían los resultados de extracción por PT-C18-SPE.

Prueba de salida. Finalmente, se evaluaron los conocimientos adquiridos por los estudiantes durante todo el proceso - además de evaluar la pertinencia de emplear una nueva metodología de preparación de muestras - desde la aplicación y diligenciamiento de un instrumento tipo cuestionario escrito que constaba de cinco preguntas, todas abiertas. Luego de aplicar esta prueba, se procedió a la evaluación con la ayuda de una rúbrica de evaluación para la presentación de los resultados.

\section{Resultados}

\section{Instrumentación y condiciones de separación}

Se desarrolló un método en modo isocrático en fase reversa para la separación de esteroides de muestras de orina. Se utilizó metanol y acetonitrilo para la determinación 
simultánea de PGN, PRe y EsD en la matriz —orina-, con el uso de una columna Phenomenex ${ }^{\otimes}$ Gemini C18 $-150 \mathrm{~mm} \times 4,60 \mathrm{~mm}, 5 \mu \mathrm{m}-$. Las condiciones se evaluaron con la fase móvil compuesta de acetonitrilo y metanol $(40+60 \mathrm{v} / \mathrm{v})$, velocidad de flujo 0,85 $\mathrm{ml} \mathrm{min}{ }^{-1}$, temperatura $40^{\circ} \mathrm{C}$, volumen de inyección $40 \mu \mathrm{l}$ y longitud de onda $260 \mathrm{~nm}$. Las proporciones de la fase móvil fueron de acetonitrilo a $55 \%$ - $45 \%$ respectivamente para obtener un ensayo rápido y simple con un tiempo de ejecución razonable, buena simetría, platos teóricos adecuados y una resolución aceptable con un tiempo de ejecución total de alrededor de 7 min (figura 3).

Figura 3

Cromatograma referente a la separación de los analitos estudiados

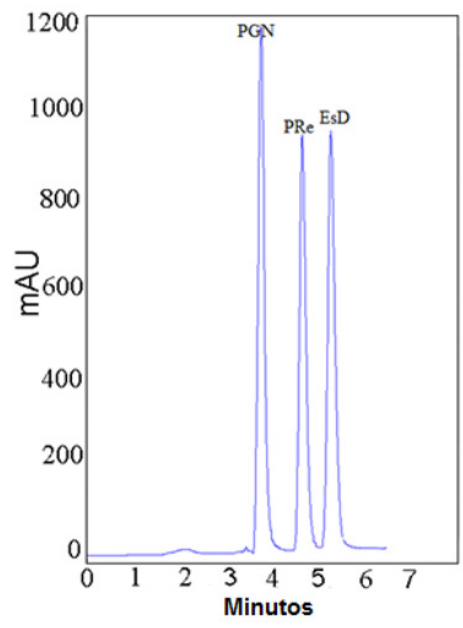

\section{Preparación de muestras empleando PT-C18-SPE}

Para optimizar la extracción de PGN, PRe y EsD en muestras de orina mediante PTC18-SPE, se evaluaron cinco parámetros tales como solvente de lavado, solvente de elución, cantidad de material $-\mathrm{C} 18-$, volumen de muestra y eluyente.

Efecto del solvente de lavado. Se realizó una etapa de lavado después de $250 \mu \mathrm{l}$ de carga de muestra. Se estudió el comportamiento de seis solventes con constante dieléctrica alta y baja: tetrahidrofurano $-150 \mu \mathrm{l}$-, agua ultrapura $-150 \mu \mathrm{l}-$, acetona $-150 \mu \mathrm{l}-$, hexano $-150 \mu \mathrm{L}-$, tolueno $-150 \mu \mathrm{l}-\mathrm{y}$ cloroformo $-150 \mu \mathrm{l}$ - . Los resultados presentaron bajas recuperaciones para todos los solventes de lavado, como se muestra en la figura 4 y se resume en la tabla 1. Estos datos mostraron que el agua eliminó la interferencia de la matriz con una baja recuperación.

Efecto del solvente de elución. Se estudió el efecto del solvente de elución para esteroides basado en el método cromatográfico desarrollado (figura 5). Se usó metanol en la primera medición y se obtuvieron bajas recuperaciones para los analitos (tabla 2). El metanol se acidificó con ácido acético y ácido fórmico en proporciones de $(9+1 \mathrm{v} / \mathrm{v})$ en ambos casos. La recuperación de los analitos permaneció constante en el caso de ácido acético mezclado con metanol como se observa en la tabla 2. Desde estos resultados, se procedió a acidular el acetonitrilo para mejorar la extracción con ácido acético en la proporción $(7+3 \mathrm{v} / \mathrm{v})$ y con ácido fórmico en la proporción $(9+1 \mathrm{v} / \mathrm{v})$. Se observó que la recuperación solo aumentó para PGN $-72,1 \%$ - con acetonitrilo/ ácido fórmico $(9+1 \mathrm{v} / \mathrm{v})$. Se concluyó que el metanol acidificado favoreció la recuperación, en la que la proporción de ácido acético se varió para una mezcla de metanol y ácido acético 
(7+3 v/v), lo que aumentó la recuperación de PGN y EsD a 71,4 \% y 73,6 \%, respectivamente, y mantuvo baja la recuperación de PRe. El metanol acidulado con ácido fórmico también se evaluó en proporción $(5+1 \mathrm{v} / \mathrm{v})$. Este es el disolvente más adecuado porque las recuperaciones de todos los analitos fueron superiores al $67 \%$, como se evidencia en la tabla 2.

\section{Figura 4}

Efecto del solvente de lavado

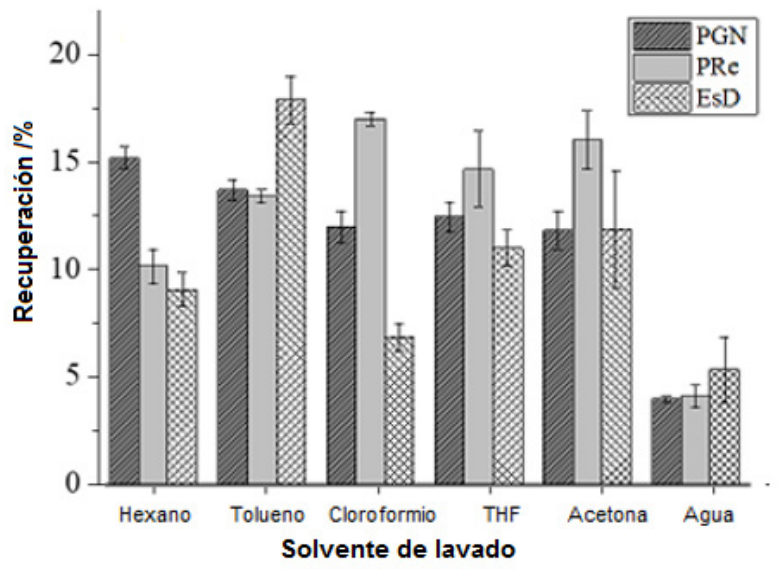

Tabla 1

Porcentajes de recuperación obtenidos por el efecto del solvente de lavado

\begin{tabular}{ccccccc}
\hline \multicolumn{7}{c}{$\%$ de recuperación } \\
\hline Solvente & THF & Agua & Acetona & Hexano & Tolueno & Cloroformo \\
\hline Analito & & & & & \\
\hline $\boldsymbol{P G N}$ & $17,20 \%$ & $5,50 \%$ & $16,30 \%$ & $12,90 \%$ & $18,90 \%$ & $16,30 \%$ \\
$\boldsymbol{P r e}$ & $20,30 \%$ & $5,60 \%$ & $22,10 \%$ & $14,00 \%$ & $18,50 \%$ & $22,10 \%$ \\
$\boldsymbol{E} \boldsymbol{S} \boldsymbol{D}$ & $15,20 \%$ & $7,10 \%$ & $16,40 \%$ & $7,40 \%$ & $24,70 \%$ & $9,50 \%$ \\
\hline
\end{tabular}

Figura 5

Efecto del solvente de elución

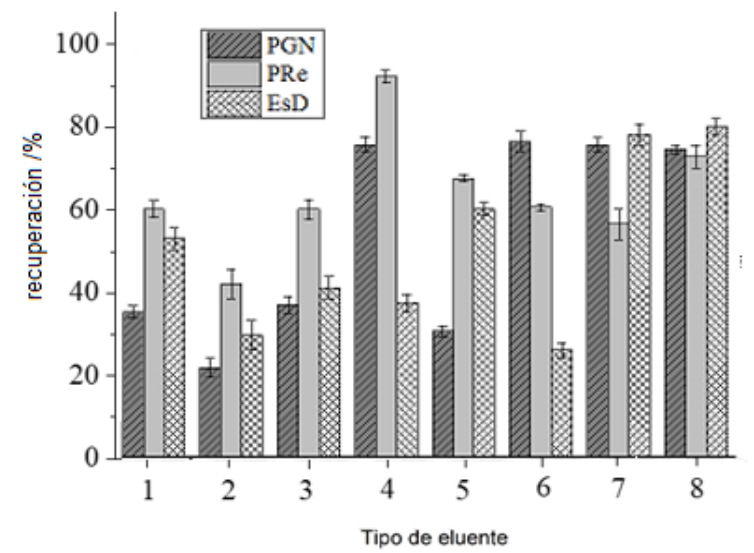

Nota: Solventes de elución: (1) metanol, (2) acetonitrilo, (3) metanol: ácido acético (9+1, v/v), (4) metanol: ácido fórmico $(9+1, \mathrm{v} / \mathrm{v}),(5)$ acetonitrilo: ácido acético $(7+3, \mathrm{v} / \mathrm{v}),(6)$ acetonitrilo: ácido fórmico $(9+1, \mathrm{v} / \mathrm{v}),(7)$ metanol: ácido acético $(7+3, \mathrm{v} / \mathrm{v}),(8)$ metanol: ácido fórmico $(5+1, \mathrm{v} / \mathrm{v})$. 
Tabla 2

Porcentajes de recuperación obtenidos por el efecto del solvente de elución

\begin{tabular}{|c|c|c|c|c|c|c|c|c|}
\hline \multicolumn{9}{|c|}{ \% de recuperación } \\
\hline Solvente & Metanol & Acetonitrilo & $\begin{array}{l}\text { Metanol: } \\
\text { Ácido } \\
\text { acético } \\
(9+1, v / v)\end{array}$ & $\begin{array}{c}\text { Metanol: } \\
\text { Ácido } \\
\text { fórmico } \\
(9+1, v / v)\end{array}$ & $\begin{array}{c}\text { Acetonitril: } \\
\text { Ácido } \\
\text { acético } \\
(7+3, v / v)\end{array}$ & $\begin{array}{c}\text { Acetonitril: } \\
\text { Ácido } \\
\text { fórmico } \\
(7+3, v / v)\end{array}$ & $\begin{array}{c}\text { Metanol: } \\
\text { Ácido } \\
\text { acético } \\
(7+3, v / v)\end{array}$ & $\begin{array}{c}\text { Metanol: } \\
\text { Ácido } \\
\text { fórmico } \\
(5+1, v / v)\end{array}$ \\
\hline \multicolumn{9}{|l|}{ Analito } \\
\hline$P G N$ & $33,20 \%$ & $25,50 \%$ & $33,20 \%$ & $71,40 \%$ & $38,90 \%$ & $72,10 \%$ & $71,40 \%$ & $72,80 \%$ \\
\hline PRe & $56,70 \%$ & $39,60 \%$ & $56,70 \%$ & $86,90 \%$ & $68,50 \%$ & $62,10 \%$ & $56,10 \%$ & $70,30 \%$ \\
\hline$E s D$ & $49,00 \%$ & $7,10 \%$ & $49,00 \%$ & $35,30 \%$ & $34,70 \%$ & $39,50 \%$ & $33,60 \%$ & $69,90 \%$ \\
\hline
\end{tabular}

Efecto del volumen de eluyente. Se evaluaron cinco parámetros para optimizar el volumen de eluyente - metanol/ácido fórmico $(7+3, v / v)$ - Según los resultados (figura 6), $50 \mu \mathrm{l}$ no son suficientes para obtener una recuperación satisfactoria. Con la excepción del EsD que presenta una recuperación aceptable de alrededor del $65 \%$, los otros analitos solo eluyeron en cantidades inferiores al $40 \%$ (tabla 3). Con $100 \mu$ l, la recuperación es ligeramente mayor para el PRe y el EsD, como se evidencia en la tabla 3. Los resultados muestran que $200 \mu \mathrm{l}$ es una cantidad apropiada de solvente de elución, ya que la recuperación se mantuvo constante para volúmenes de $250 \mu \mathrm{l}$ de disolvente de elución (tabla 3).

\section{Figura 6}

Efecto del volumen de solvente de elución

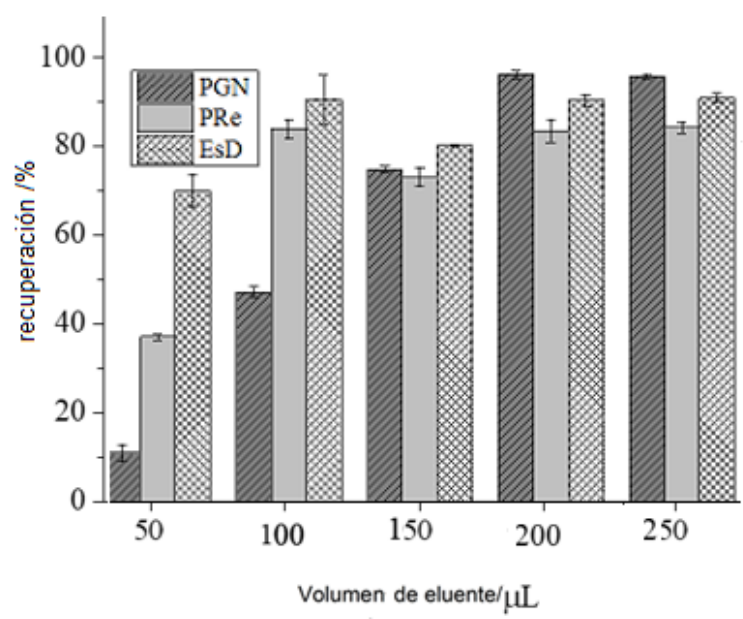

Efecto de la cantidad de C18. Se evaluaron algunas cantidades de C18: 10, 20, 30 y 40 $\mathrm{mg}$. En la figura 7 puede verse que la cantidad de $10 \mathrm{mg}$ y $20 \mathrm{mg}$ fue pobre para recuperar todos los analitos, para las que la recuperación permaneció por debajo del $50 \%$. Con $30 \mathrm{mg}$, la recuperación mejoró sustancialmente para PGN y EsD, ya que alcanzó recuperaciones cercanas al $70 \%$ y $60 \%$ respectivamente. $4 \mathrm{mg}$ resultó ser la cantidad apropiada para la extracción de esteroides en la orina porque los resultados muestran que PGN alcanzó una recuperación cercana al 90 \%, para PRe 78,4 \% y para EsD 85,0 \%. El valor p (tabla 3) confirma estadísticamente que la recuperación según la cantidad de material es relevante y que el C18 está perfectamente adaptado a la técnica PT-SPE. 
Tabla 3

Porcentajes de recuperación obtenidos por el efecto del volumen de eluyente

\begin{tabular}{cccccc}
\hline \multicolumn{7}{c}{ \% de recuperación } \\
\hline Volumen & $\mathbf{5 0}$ & $\mathbf{1 0 0}$ & $\mathbf{1 5 0}$ & $\mathbf{2 0 0}$ & $\mathbf{2 5 0}$ \\
\hline Analito & & & & \\
\hline $\boldsymbol{P G N}$ & $16,30 \%$ & $25,50 \%$ & $70,40 \%$ & $89,60 \%$ & $89,60 \%$ \\
$\boldsymbol{P R} \boldsymbol{e}$ & $39,80 \%$ & $68,70 \%$ & $68,70 \%$ & $78,40 \%$ & $78,40 \%$ \\
$\boldsymbol{E} \boldsymbol{D} \boldsymbol{D}$ & $65,10 \%$ & $75,40 \%$ & $75,40 \%$ & $84,90 \%$ & $84,90 \%$ \\
\hline
\end{tabular}

Figura 7

Efecto de la cantidad de C18

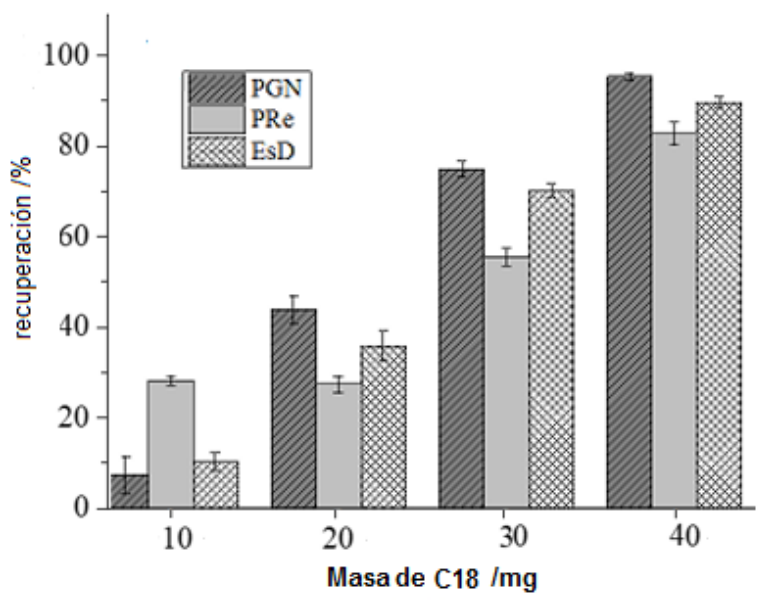

Figura 8

Efecto del volumen de muestra

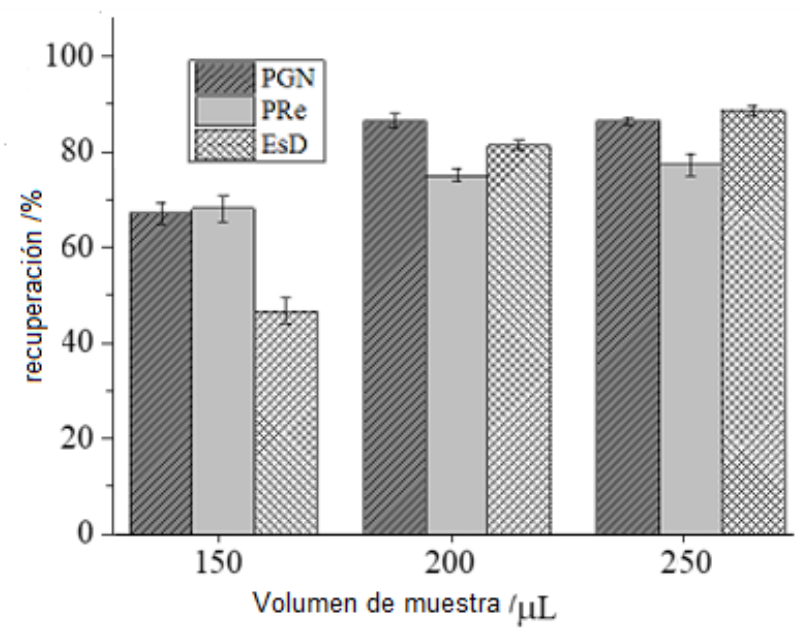

Efecto del volumen de muestra. El efecto del volumen de la muestra se estudió cargando PT-C18-SPE con 75, 150, 200 y $250 \mu$ de las muestras de orina fortificadas. Los resultados mostraron buenas recuperaciones al emplear $200 \mu \mathrm{l}$ que muestran una recuperación de 90,4 \% para PGN, 78,4 \% para PRe y 84,9 \% para EsD. Por su parte, $250 \mu$ l muestran una recuperación de $90,4 \%$ para $\mathrm{PGN}, 80,7 \%$ para PRe y 92,6 \% para EsD (figura 8). 


\section{Validación del método}

Se analizaron las muestras en blanco, enriquecidas y reales. Los datos obtenidos para la selectividad no mostraron la presencia de interferencia en el mismo tiempo de retención de analitos, como se muestra en la figura 9 -línea roja-. La tabla 4 muestra sus coeficientes de correlación $[\mathrm{r}]$, coeficiente de determinación $\left[\mathrm{R}^{2}\right]$, rango lineal, pendiente, porcentaje de desviación estándar relativa [RSD \%] y las ecuaciones lineales, en la tabla 5 se observa el LOQ y porcentaje de error relativo [RE \%] para el método utilizado, que están de acuerdo con las directrices para la validación de los métodos analíticos convencionales. Los valores RSD \% y $\mathrm{RE} \%$ para la precisión y exactitud de los estudios intra- e interdiarios fueron inferiores al $5 \%$ (tabla 6). El método demostró ser robusto bajo las variaciones estudiadas (tabla 7).

\section{Figura 9}

Cromatogramas referentes a la inyección de blanco -línea negra-, muestras con patrones analíticos -línea azul-, muestra real -línea roja-

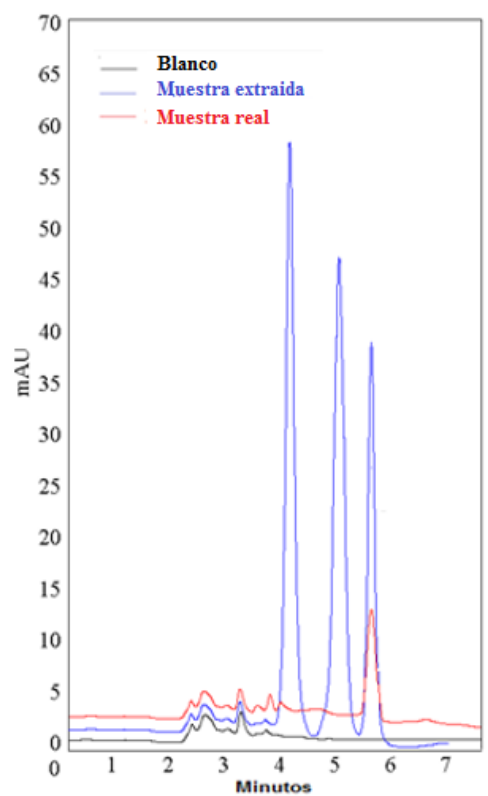

Tabla 4

Linealidad del método analítico en muestras de orina fortificadas.

\begin{tabular}{|c|c|c|c|c|}
\hline \multicolumn{5}{|c|}{ Linealidad } \\
\hline Analitos & Ecuación linear ${ }^{a}$ & $r$ & Intervalo (ng ml $\left.{ }^{-1}\right)$ & $\operatorname{RSD}^{b}(\%)$ \\
\hline PGN & $y=112.48 x+18207$ & 0.9985 & $16-1200$ & 2.49 \\
\hline PRe & $y=292.08 x+96726$ & 0.9956 & $16-1200$ & 3.89 \\
\hline EsD & $y=427.20 x+15250$ & 0.9988 & $16-1200$ & 2.08 \\
\hline
\end{tabular}

Nota: ${ }^{\text {a }}$ Las curvas de calibración se determinaron por triplicado $(\mathrm{n}=3)$ para concentraciones de 100, 300, 500, 1000, 1500 y 2000 ng ml-1; y = ax + b, donde y es el área del pico del analito, a es la pendiente, b es la intersección y x es la concentración de la solución medida ng ml-1.

${ }^{\mathrm{b}} \mathrm{RSD}=$ desviación estándar relativa de la pendiente de la curva de calibración 
Tabla 5

Límite de cuantificación del método analítico en muestras de orina fortificadas

\begin{tabular}{|c|c|c|c|c|}
\hline \multicolumn{5}{|c|}{ Límite de cuantificación } \\
\hline Analitos & Nominal (ng ml ${ }^{-1}$ ) & Analizado $\left(\mathrm{ng} \mathrm{ml}^{-1}\right)$ & $\operatorname{RSD}^{a}(\%)$ & $\operatorname{RE}^{b}(\%)$ \\
\hline PGN & 16.00 & 16.84 & 1.61 & 1.60 \\
\hline PRe & 16.00 & 16.39 & 1.24 & 1.59 \\
\hline EsD & 16.00 & 16.24 & 1.17 & 1.36 \\
\hline
\end{tabular}

Nota: ${ }^{\text {a }} \mathrm{RSD}$ = desviación estándar relativa del límite de cuantificación.

${ }^{\mathrm{b}} \mathrm{RE}=$ error relativo con un promedio de seis repeticiones.

Tabla 6

Precisión y exactitud del método analítico para la determinación de esteroides

\begin{tabular}{|c|c|c|c|c|c|c|c|c|c|}
\hline Analitos & & $P G N$ & & & PRe & & & $E s D$ & \\
\hline $\begin{array}{c}\text { Concentración } \\
\text { Nominal }\left(\mathrm{ng} \mathrm{mL}^{-1}\right)\end{array}$ & 40 & 200 & 800 & 40 & 200 & 800 & 40 & 200 & 800 \\
\hline $\begin{array}{l}\text { Concentración analiza- } \\
\qquad \mathrm{da}\left(\mathrm{ng} \mathrm{mL} \mathrm{m}^{-1}\right)\end{array}$ & 38.11 & 205.58 & 795.73 & 37.74 & 203.00 & 801.07 & 52.24 & 187.31 & 797.69 \\
\hline Precisión (RSD ${ }^{\mathrm{b}}, \%$ ) & 0.84 & 0.34 & 0.34 & 1.14 & 7.83 & 1.92 & 1.08 & 1.57 & 2.55 \\
\hline Error relativo $\left(\mathrm{RE}^{\mathrm{c}}, \%\right)$ & -4.71 & 2.79 & 0.53 & -5.66 & 1.50 & 0.13 & 3.59 & -1.35 & -0.29 \\
\hline $\begin{array}{l}\text { Concentración analiza- } \\
\qquad \mathrm{da}\left(\mathrm{ng} \mathrm{mL} \mathrm{m}^{-1}\right)\end{array}$ & 35.80 & 217.06 & 834.13 & 31.29 & 223.97 & 796.85 & 48.32 & 192.95 & 816.59 \\
\hline Precisión $\left(\mathrm{RSD}^{\mathrm{b}}, \%\right)$ & 1.50 & 0.61 & 2.38 & 2.08 & 1.25 & 1.08 & 0.76 & 1.76 & 3.19 \\
\hline Error relativo $\left(\mathrm{RE}^{\mathrm{c}}, \%\right)$ & -5.25 & 8.53 & 4.27 & -1.79 & 1.99 & -0.39 & 2.04 & -3.52 & 2.07 \\
\hline
\end{tabular}

Nota: ${ }^{\text {a }} \mathrm{n}=$ número de repeticiones.

${ }^{\mathrm{b}} \mathrm{RSD}(\%)$ = desviación estándar relativa.

${ }^{\mathrm{c}} \mathrm{RE}(\%)=$ error relativo.

Tabla 7

Test de robustez para análisis de esteroides en orina

\begin{tabular}{|c|c|c|c|c|c|c|c|c|c|c|}
\hline Analit & & & $P G N$ & & & $P R$ & & & $E s D$ & \\
\hline Variaciones & & $\begin{array}{l}{ }^{a} R E \\
\text { (\%) }\end{array}$ & $\begin{array}{c}{ }^{6} \text { RSD } \\
\text { (\%) }\end{array}$ & ${ }^{c} p$-value & $\begin{array}{l}{ }^{a} R E \\
\text { (\%) }\end{array}$ & $\begin{array}{c}{ }^{\prime} \text { RSD } \\
\text { (\%) }\end{array}$ & 'p-value & $\begin{array}{l}{ }^{a} R E \\
(\%)\end{array}$ & $\begin{array}{c}{ }^{\mathrm{D}} \text { RSD } \\
\text { (\%) }\end{array}$ & 'p-value \\
\hline & 0.75 & 6.44 & 3.55 & & 1.66 & 0.84 & & 2.31 & 3.55 & \\
\hline & 0.85 & 0.98 & 0.96 & 0.41 & 0.18 & 0.38 & 0.10 & -1.39 & 1.44 & 0.07 \\
\hline (mI min ) & 0.95 & 2.71 & 1.73 & & 1.92 & 2.83 & & 1.52 & 2.70 & \\
\hline Composi- & $40: 60$ & 0.98 & 0.72 & & 1.60 & -0.34 & & 1.33 & -0.66 & \\
\hline $\begin{array}{l}\text { ción } \\
\text { Fase }\end{array}$ & 45:55 & 0.88 & 2.49 & 0.25 & 3.95 & 1.82 & 0.43 & 2.52 & 1.53 & 0.05 \\
\hline Móvil & $50: 50$ & 3.84 & 2.60 & & 1.58 & 1.52 & & 2.06 & 3.22 & \\
\hline
\end{tabular}




\begin{tabular}{ccccccccccc} 
Inyección & 40.00 & 0.90 & 2.55 & & -0.84 & 2.52 & & 0.93 & 1.62 & \\
$\begin{array}{c}\text { de mues- } \\
\text { tra }\end{array}$ & 45.00 & 0.90 & 2.49 & 0.31 & 3.95 & 1.82 & 0.47 & 3.95 & 1.53 & 0.05 \\
$\quad$ & & & & & & & & & & \\
$\boldsymbol{\mu l}$ & 50.00 & 3.40 & 1.72 & & 2.64 & 1.25 & & 3.76 & 1.64 & \\
\hline
\end{tabular}

Nota: ${ }^{\text {a }}$ RSD (\%) = desviación estándar relativa.

${ }^{\mathrm{b}} \mathrm{ER}(\%)=$ error relativo.

${ }^{\mathrm{c}}$ Nivel de significancia establecido en $\mathrm{p}<0,05$.

\section{Intervención didáctica}

Se realizó la estrategia de intervención didáctica con un enfoque basado en los TPL aplicados a química. Desde la consulta guiada de los experimentos que involucraron y desafiaron a los estudiantes en todos los pasos del proceso experimental -búsqueda de literatura, planificación y realización de su propio experimento, y analizando y discutiendo datos experimentales-, se generó una mejor comprensión de los procedimientos experimentales (Supasorn, 2012). Por otro lado, las dos primeras fases de la intervención se guiaron para mejorar los modelos mentales de los estudiantes de los conceptos correspondientes a la práctica pretexto. Esto ayudó a los estudiantes a comprender los conceptos clave en química, especialmente a un nivel submicroscópico. En ese orden de ideas, las habilidades procedimentales de los instructores de laboratorio en la preparación de muestras, quienes simplemente mecanizan el desarrollo de los experimentos, pueden ser deficientes para la comprensión de los conceptos de laboratorio a un nivel submicroscópico. Con base en lo anterior, esta intervención, mediante la práctica continua de experimentos, y la indagación mejorarían las habilidades de investigación científica de los estudiantes en cuatro fases:

1. Determinación de las ideas previas de los estudiantes mediante un instrumento para conocer qué sabían sobre la temática a trabajar.

2. Exposición sobre extracción en fase sólida, técnicas miniaturizadas y metodologías de cuantificación por HPLC.

3. Práctica de laboratorio titulada Extracción en fase sólida para la cuantificación de fármacos en muestras complejas y realización de la práctica de laboratorio Cuantificación de esteroides por HPLC a partir de muestras de orina empleando extracción en fase sólida adaptada en puntera.

4. Evaluación final, con la cual se comparó el cambio conceptual, respecto a la temática de SPE, con respecto al test de ideas previas.

Prueba de ideas previas. los resultados expuestos en la figura 11 revelan que: En el ítem 1, nueve estudiantes señalaron lo que sucede al separar los componentes de un compuesto y de una mezcla. Diez estudiantes presentaron ideas alternativas al no tener claridad acerca de la separación de mezclas y compuestos.

En el ítem 2, seis estudiantes señalaron las diferencias entre un sistema binario y un sistema terciario, al aludir a las propiedades fisicoquímicas que caracterizan y definen cada sistema. Cinco estudiantes no contestaron la pregunta, mientras que diez estudiantes no supieron explicar ni definir las características de un sistema binario o terciario, ya 
que mostraron concepciones erróneas, refiriéndose más a propiedades extensivas que no definían nada de lo preguntado.

En el ítem 3, para diez estudiantes el proceso de adsorción es el que se lleva a cabo al escribir sobre el papel. Supieron interpretar la figura. Cinco estudiantes complementaron sus respuestas diciendo que la tinta penetraba los poros del papel por lo que no evidencian un cambio conceptual, debido a la escasa diferenciación entre procesos a nivel microscópico y a nivel macroscópico.

En el ítem 4, quince estudiantes indicaron correctamente el orden en que debían ubicar las sustancias de acuerdo con la instrucción dada, por lo que se puede afirmar que los conceptos de polaridad, fuerzas intermoleculares y eluotropía están claros para la mayoría de la población muestreada. Cinco estudiantes erraron en el orden al situar las sustancias.

Finalmente, en el último ítem, diez estudiantes afirmaron que la extracción líquidolíquido es el método más apropiado. Once señalaron la extracción en fase sólida, la cual era la respuesta más apropiada, debido a la necesidad de mejorar la recuperación del analito, desde sus posibilidades de cuantificación.

\section{Figura 11}

Resultados test de ideas previas

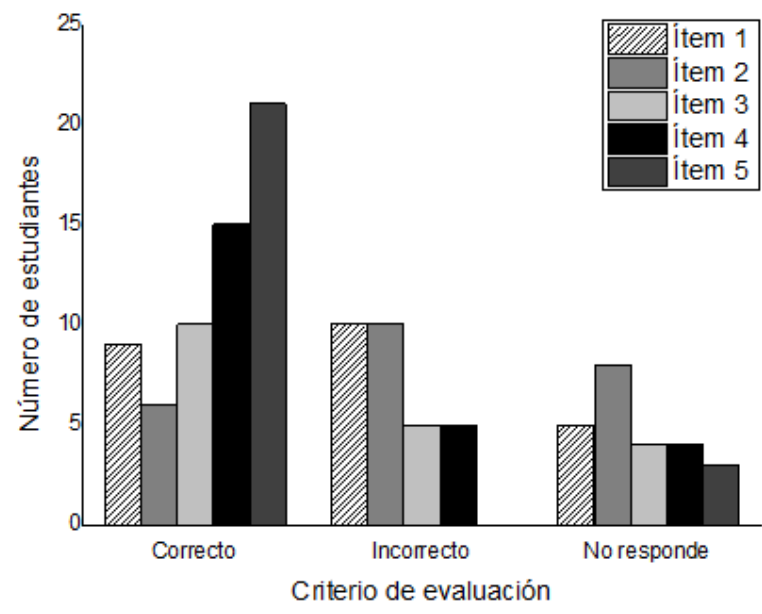

Trabajos prácticos de laboratorio. La práctica 1 consistió en realizar una extracción en fase sólida. Los estudiantes tenían los objetivos, junto con el material, el método y una solución a la práctica de laboratorio, ya que se les facilitó todo el procedimiento. Sin embargo, al final se les dejaron unas preguntas que debían responder según sus conocimientos o su motivación por encontrar una solución. Así, los ítems que fueron tenidos en cuenta para llevar a cabo la práctica fueron:

Parámetros de optimización. En este ítem se evaluaba la optimización de los parámetros de preparación de muestras. Como se puede observar en la tabla 8, los cinco grupos participantes cumplieron con su presentación en el informe.

Porcentaje de recuperación. Este ítem evaluaba la presentación en el informe de la relación que permitiera evidenciar que los estudiantes habían comprendido el concepto de porcentaje de recuperación y su relación con la obtención de los analitos. En la tabla 8 
se observa que los cinco grupos que participaron en el laboratorio cumplieron con este requisito para la presentación en el informe.

Procesos de extracción. Se evaluaba la presentación de los procesos de extracción en diagrama de bloque, con el fin de que los estudiantes evidenciaran que habían comprendido el funcionamiento y el proceso que sigue la extracción en fase sólida. De los siete grupos que participaron en el laboratorio, seis cumplieron el requisito.

Cuestionario. Se evaluaba el desarrollo de un cuestionario que constaba de cuatro preguntas sobre conceptos como polaridad, elución, percolación y adsorbente y la diferencia entre técnicas de extracción miniaturizadas y de extracción convencionales. De los cinco grupos que participaron en el laboratorio, dos cumplieron al tenerlo totalmente bien, o con una respuesta mal. Tres grupos fallaron en más de dos de las cuatro preguntas. Las preguntas en las que los estudiantes presentaron más problemas fueron la $3-$ ya que se evidenció que no tenían claro el concepto de polaridad - y la 4 - en donde no se tuvo claro el concepto de percolación-.

Tabla 8

Rúbrica de evaluación para el informe de laboratorio "Extracción en fase sólida para la cuantificación de fármacos en muestras complejas"

\begin{tabular}{|c|c|c|c|c|c|c|}
\hline \multirow{3}{*}{ Ítem } & \multirow{3}{*}{ ¿Qué evalúa? } & \multicolumn{2}{|c|}{ Criterios de evaluación } & \multirow{2}{*}{\multicolumn{3}{|c|}{$\begin{array}{l}\text { Puntuación } \\
\text { Frecuencia }\end{array}$}} \\
\hline & & \multirow[b]{2}{*}{ Correcto } & \multirow[b]{2}{*}{ Incorrecto } & & & \\
\hline & & & & Correcto & Incorrecto & $\begin{array}{c}\text { No } \\
\text { realizado }\end{array}$ \\
\hline $\begin{array}{l}\text { Parámetros de } \\
\text { optimización }\end{array}$ & $\begin{array}{l}\text { Adecuada } \\
\text { presentación de } \\
\text { ecuaciones de } \\
\text { recuperación de } \\
\text { analitos. }\end{array}$ & $\begin{array}{l}\text { Presenta de } \\
\text { forma adecuada } \\
\text { las ecuaciones } \\
\text { de recuperación } \\
\text { de los analitos } \\
\text { correctamente }\end{array}$ & $\begin{array}{l}\text { Presenta de } \\
\text { forma adecuada } \\
\text { las ecuaciones } \\
\text { de recuperación } \\
\text { de los analitos } \\
\text { incorrectamente }\end{array}$ & $7 / 7$ & $0 / 7$ & $0 / 7$ \\
\hline $\begin{array}{l}\text { Porcentaje de } \\
\text { recuperación }\end{array}$ & $\begin{array}{l}\text { Presentación } \\
\text { del porcentaje } \\
\text { de recuperación } \\
\text { estadístico. }\end{array}$ & $\begin{array}{l}\text { Evidencia el } \\
\text { porcentaje de } \\
\text { recuperación } \\
\text { estadístico. }\end{array}$ & $\begin{array}{l}\text { No evidencia el } \\
\text { porcentaje de } \\
\text { recuperación } \\
\text { estadístico. }\end{array}$ & $6 / 7$ & $1 / 7$ & $0 / 7$ \\
\hline $\begin{array}{l}\text { Procesos de } \\
\text { extracción }\end{array}$ & $\begin{array}{l}\text { Presentación de } \\
\text { los procesos de } \\
\text { extracción en } \\
\text { diagramas de } \\
\text { bloque. }\end{array}$ & $\begin{array}{l}\text { El diagrama de } \\
\text { bloque presenta } \\
\text { una secuencia } \\
\text { coherente de } \\
\text { los procesos de } \\
\text { extracción. }\end{array}$ & $\begin{array}{l}\text { El diagrama de } \\
\text { bloque no presenta } \\
\text { una secuencia } \\
\text { coherente de } \\
\text { los procesos de } \\
\text { extracción. }\end{array}$ & $6 / 7$ & $1 / 7$ & $0 / 7$ \\
\hline Cuestionario & $\begin{array}{l}\text { Desarrollo del } \\
\text { cuestionario. }\end{array}$ & $\begin{array}{l}\text { Desarrolla el } \\
\text { cuestionario de } \\
\text { forma correcta } \\
\text { o máximo con } \\
\text { un ítem errado. }\end{array}$ & $\begin{array}{l}\text { Desarrolla el } \\
\text { cuestionario de } \\
\text { forma incorrecta o } \\
\text { con más de un ítem } \\
\text { errado. }\end{array}$ & $4 / 7$ & $3 / 7$ & $0 / 7$ \\
\hline
\end{tabular}

Se evidenciaron resultados propios de una práctica de tipo expositiva en la que los estudiantes son poco exigidos. El principal inconveniente de esta modalidad de prácticas es su ineficacia de cara al cambio conceptual del alumnado, ya que solo se requieren procesos cognitivos de bajo orden y los estudiantes pasan más tiempo determinando si han conseguido o no los resultados correctos que planificando y organizando el experimento (Chen et al., 2016). 
Se realizó una segunda práctica, la cual tenía como finalidad observar el desenvolvimiento de los estudiantes en un contexto en el cual tenían que alcanzar un objetivo, al indicar el instrumental a emplear y la metodología a desarrollar para resolver un problema con una solución abierta.

A continuación, se presentan los ítems tenidos en cuenta para la evaluación con su respectivo resultado.

Diseño experimental. Se evaluaba la relación entre las variables y el procedimiento que debían seguir los estudiantes para la preparación de la muestra. Para ello, se entregaba un problema y se les daba a los estudiantes un posible orden que podrían utilizar, pero que ellos podían escoger. Los estudiantes entregaban este ítem al iniciar la práctica. Los resultados que se obtuvieron fueron que dos de los siete grupos tenían bien el procedimiento y la forma apropiada de ejecutar la técnica de preparación de muestras.

Manejo de datos. Este ítem se subdividió en otros para su evaluación:

1. Determinación del porcentaje de recuperación. Este ítem evaluaba que los estudiantes determinaran el porcentaje de recuperación por medio del método de adición estándar. En este apartado, se encontraron dificultades en los estudiantes en la medida que algunos de ellos no tenían habilidades en el manejo de Excel. Tan solo tres de los siete grupos de trabajo obtuvieron de forma correcta este cálculo, lo que evidencia la problemática relacionada con la aplicación de ecuaciones y fórmulas.

2. Manejo estadístico. Se evaluaba que los estudiantes aplicaran de forma correcta la estadística inferencial para el manejo de datos, con el fin de obtener buenos parámetros de reproducibilidad, precisión, exactitud y robustez. De los siete grupos, cinco lo hicieron de forma correcta.

3. Análisis de resultados. Se evaluaba la interpretación que los estudiantes le daban a los resultados que habían obtenido. En este caso, era fundamental que los estudiantes mencionaran los parámetros de precisión y exactitud, al igual que comentaran cuáles son las condiciones óptimas de preparación de muestras para obtener recuperaciones cercanas al $100 \%$. También era importante mencionar el por qué en los cromatogramas aparecían otros picos y su interpretación. De los siete grupos, tan solo dos hicieron un adecuado análisis. Los restantes cinco grupos, aunque mencionaban para ellos qué eran los otros picos, no podían hacer un análisis pertinente, debido a que sus resultados no se habían realizado de forma correcta.

Los datos obtenidos evidenciaron la presencia de falencias en cuanto a la presentación de los resultados, lo cual impide realizar un adecuado análisis. Aunque esta práctica era de tipo intermedio, se encontraron resultados poco satisfactorios. Se cree que esto sucedió porque los estudiantes están acostumbrados a emplear procesos cognitivos de bajo orden, pero cuando se enfrentan a prácticas en las que se requieren procesos cognitivos de alto orden, se presentan confusiones, lo que deriva en resultados errados.

Prueba de salida. Teniendo en cuenta el trabajo experimental y el trabajo disciplinar, los estudiantes del grupo objetivo contaban con las herramientas suficientes para realizar la prueba de salida cuyos resultados se muestran en la figura 12. Se evidenció un aumento significativo de las habilidades de los estudiantes para responder a las situaciones 
problemáticas con los conocimientos adquiridos de SPE.

La primera situación problema indagaba en los estudiantes la identificación de procesos de preparación de muestras específicos para un procedimiento en especial. Diecinueve estudiantes respondieron de forma incorrecta, puesto que no había claridad al diferenciar exclusión molecular de extracción molecular, que es un término no muy empleado. Esta confusión de conceptos derivó en las respuestas erradas de los estudiantes.

Para el ítem 2, nueve estudiantes respondieron de forma correcta al identificar el tipo de solvente que se debe utilizar para un sistema, para el que los materiales adsorbentes diferían mucho en su polaridad. Catorce estudiantes ignoraron la serie eluotrópica y se inclinaron a responder bajo puntos de vista de soluciones orgánicas, sin especificar la polaridad o el tipo de elución precisa para el sistema de extracción expuesto en la pregunta.

En el ítem 3 se preguntó por las ventajas de emplear solventes acidulados. Dieciséis estudiantes acertaron en su respuesta y ocho respondieron de forma incorrecta, lo cual evidencia que no hay claridad en los conceptos de elución y percolación.

En el ítem 4, ocho estudiantes respondieron de forma correcta, nueve de forma incorrecta y siete no respondieron. En este punto se evidenció que solo quienes tenían claridad sobre el sistema presentado pudieron sugerir de forma coherente cómo variar la polaridad de la fase móvil para eluir solutos con diferente polaridad.

Finalmente, en el ítem 5 se indagó si el estudiante evidenciaba la diferencia entre adsorción y absorción. Dieciséis estudiantes contestaron de forma acertada, lo que confirma que hubo un adecuado cambio conceptual.

Figura 12

Resultados prueba de salida

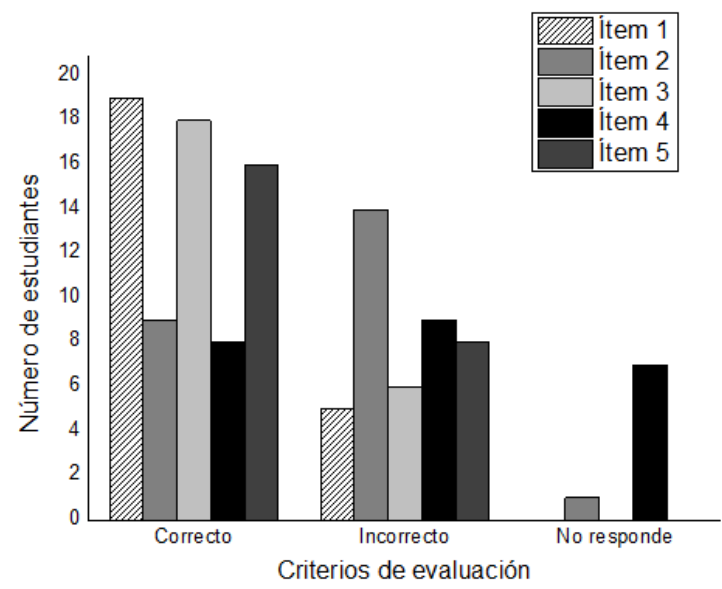

\section{Conclusiones}

En este trabajo se desarrolló un método para multideterminación de esteroides en muestras de orina empleando HPLC-DAD. Tanto el método como la técnica demostraron 
ser económicos, rápidos y fáciles de operar. Además, la PT-C18-SPE es un procedimiento ambientalmente correcto, al utilizar cantidades mínimas de solvente orgánico $-\mu \mathrm{l}-$ en el proceso de optimización de la preparación de la muestra con excelente eficiencia de extracción -alrededor del $90 \%$ - para PGN y EsD, y un valor de $85 \%$ para PRe. Finalmente, la metodología mostró valores aceptables en el proceso de validación.

A través de los trabajos prácticos de laboratorio, se promovió la adquisición de habilidades para métodos de preparación de muestras no convencionales, específicamente de extracción en fase sólida. Según las ideas previas de los estudiantes y en comparación con la prueba de salida en el grupo objetivo, se concluye que hubo un cambio conceptual significativo en lo referente a la diferenciación entre absorción y adsorción. Al comparar las ideas previas y la evaluación final del proceso en el cual se desarrolló este trabajo, se evidenció que el estudiante se familiarizó y amplió su conocimiento disciplinar al apropiarse de "nuevos métodos" de preparación de muestras como lo son los métodos miniaturizados, lo que permitió un aumento significativo en el lenguaje químico, así como en la comprensión de conceptos estructurantes para su buen desempeño como profesional de la química.

No se evidenció una aproximación clara por parte de los estudiantes para relacionar los métodos cromatográficos con un contexto particular, pero sí se obtuvieron resultados favorables en la práctica en donde se requerían procesos cognitivos de bajo orden. Por otra parte, en la práctica de nivel de complejo se obtuvieron resultados muy bajos, ya que se requerían procesos cognitivos de alto orden. Dados los resultados poco favorables que se tuvieron en la práctica con procesos cognitivos de alto orden, es necesario redimensionar el papel del profesor en el sentido de hacerlo más activo, en la medida que acompañe y guíe permanentemente el proceso experimental.

\section{Referencias}

Alcántara-Durán, J., Moreno-González, D., Gilbert-López, B., Molina-Díaz, A., \& García-Reyes, J. (2018). Matrix-effect free multi-residue analysis of veterinary drugs in food samples of animal origin by nanoflow liquid chromatography high resolution mass spectrometry. Food Chemistry, 245, 29-38. http://dx.doi.org/10.1016/j. foodchem.2017.10.083

Amiri, A., \& Ghaemi, F. (2017). Microextraction in packed syringe by using a threedimensional carbon nanotube/carbon nanofiber-graphene nanostructure coupled to dispersive liquid-liquid microextraction for the determination of phthalate esters in water samples. Microchimica Acta, 184(10), 3851-3848. https://doi.org/10.1007/s00604017-2416-8

Angulo, D., Andrade, R., Santos, R., Carneiro, B., Avellar, F., \& Bastos, K. (2018). Pipettetip solid-phase extraction using polypyrrole as efficient adsorbent for extraction of avermectins and milbemycins in milk. Analytical and Bioanalytical Chemistry, 410(14), 3361-3374. https://doi.org/10.1007/s00216-018-1031-9

Bostan, I., Beloiu, R., \& Bizon, N. (2015). Aprendiendo divisores digitales de frecuencia a través de actividades prácticas de laboratorio. Procedia - Social and Behavioral Sciences, 180, 1014-1021. https://doi.org/10.1016/j.sbspro.2015.02.195 
Chen, W., Shah, U., \& Brechtelsbauer, C. (2016). The discovery laboratory - A studentcentred experiential learning practical: Part I - Overview. Education for Chemical Engineers, 17, 44-53. https://doi.org/10.1016/j.ece.2016.07.005

Chiesa, L., Nobile, M., Biolatti, B., Pavlovic, R., Panseri, S., Cannizzo, F., \& Arioli, F. (2016). Detection of selected corticosteroids and anabolic steroids in calf milk replacers by liquid chromatography-electrospray ionization-tandem mass spectrometry. Food Control, 61, 196-203. http://dx.doi.org/10.1016/j.foodcont.2015.09.028

Dănescu, E. (2015). Intercultural Education from the Perspective of Training Didactic Competences. Procedia - Social and Behavioral Sciences, 180, 537-542. https://doi. org/10.1016/j.sbspro.2015.02.156

Dönmez, N., \& Ayas, A. (2010). Common misconceptions in nuclear chemistry unit. Procedia -Social and Behavioral Sciences, 2(2)1432-1436.

Franco, R., Velasco, M., \& Riveros, C. (2017). Los trabajos prácticos de laboratorio en la enseñanza de las ciencias: tendencias en revistas especializadas: 2012-2016. Tecné, Episteme y Didaxis, TED, (41), 37-56.

Gañán, J., Morante-Zarcero, S., Pérez-Quintanilla, D., Marina, M., \& Sierra, I. (2016). One-pot synthesized functionalized mesoporous silica as a reversed-phase sorbent for solidphase extraction of endocrine disrupting compounds in milks. Journal of Chromatography A, 1428, 228-235. http://dx.doi.org/10.1016/j.chroma.2015.08.063

Ghibaudi, E., Roletto, E., \& Regis, A. (2016). Didattica della chimica e trasposizione didattica. Parte prima - I fondamenti teorici di una prassi. Perspectives in Science, 10, 19-27. https://doi.org/10.1016/j.pisc.2016.03.010

Hassan, E., Talbi, M., \& Radid, M. (2015). Teaching and Learning of Experimental Science: The Case of Chemistry in Secondary Qualifying Morocco. Procedia - Social and Behavioral Sciences, 191, 2246-2249. https://doi.org/10.1016/j.sbspro.2015.04.338

Hernández, G. (2012). Enseñanza experimental. ¿Cómo y para qué? Educación Química, 23, 92-95.

Jiang, Y., Ma, P., Li, X., Piao, H., Li, D., Sun, Y., Wang, X., \& Song, D. (2018). Application of metalorganic framework MIL-101(Cr) tomicroextraction in packed syringe for determination of triazineherbicides in corn samples by liquid chromatography-tandem mass spectrometry. Journal of Chromatography A, 1574, 36-41. https://doi.org/10.1016/j. chroma.2018.09.008

Kampelmann, S., Kaethler, M., \& Vickery, A. (2018). Curating complexity: An artful approach for real-world system transitions. Environmental Innovation and Societal Transitions, 27, 59-71. https://doi.org/10.1016/j.eist.2017.10.005

Li, N., Yang, X., Nian, L., Wang, Z., Lei, L., Wang, K., Zhang, H., Yu, A., \& Zhang, Z. (2015). Determination of steroid hormones in milk using aqueous two-phase extraction coupled to liquid chromatography. Analytical Methods, 7(6), 2514-2522. https://doi.org/10.1039/ c4ay03036b

Liu, H., Lin, T., Cheng, X., Li, N., Wang, L., \& Li, Q. (2017). Simultaneous determination 
of anabolic steroids and $\beta$-agonists in milk by QuEchERS and ultra-high performance liquid chromatography tandem mass spectrometry. Journal of Chromatography $B, 1043$, 176-186. http://dx.doi.org/10.1016/j.jchromb.2016.08.016

Nacimento do, T., Avellar, F., Carneiro, B., \& Bastos, K. (2018). Efficient removal of antiinflammatory phenylbutazone from an aqueous solution employing a composite material based on poly(aniline-co-pyrrole)/multi-walled carbon nanotubes. New Journal of Chemistry, 42(9), 7030-7042. https://doi.org/10.1039/c8nj00861b

Nezhadali, A., Es'haghi, Z., \& Khatibi, A. (2016). Selective extraction of progesterone hormones from environmental and biological samples using a polypyrrole molecularly imprinted polymer and determination by gas chromatography. Analytical Methods, 8(8), 1813-1827. https://doi.org/10.1039/c5ay02174j

Özmen,H., Demircioewag囚lu,H., \&Demirciog冈lu, G. (2009). Theeffects of conceptualchange texts accompanied with animations on overcoming 11th grade students' alternative conceptions of chemical bonding. Computers \& Education, 52, 681-695.

Pelit, F., Pelit, L., Dizdaş, T., Aftafa, C., Ertaş, H., Yalçınkaya, E., Türkmen, H., \& Ertaş, F. (2015). A novel polythiophene - ionic liquid modified clay composite solid phase microextraction fiber: preparation, characterization and application to pesticide analysis. Analytica Chimica Acta, 859, 37-45. http://dx.doi.org/10.1016/j.aca.2014.12.043

Ponzetto, F., Boccard, J., Baume, N., Kuuranne, T., Rudaz, S., Saugy, M., \& Nicoli, R. (2017). High-resolution mass spectrometry as an alternative detection method to tandem mass spectrometry for the analysis of endogenous steroids in serum. Journal of Chromatography B, 1052, 34-42. http://dx.doi.org/10.1016/j.jchromb.2017.03.016

Qing, Z., Nia, S., \& Hong, T. (2010). Developing critical thinking disposition by task-based learning in chemistry experiment teaching. Procedia - Social and Behavioral Sciences, 2, 4561-4570.

Razmkhah, K., Sereshti, H., Soltani, S., \& Nodeh, H. (2018). Extraction and determination of three steroid molecules in milk using functionalized magnetic carbon nanotubebased solid phase extraction coupled with HPLC. Food Analytical Methods, 11(11), 3179-3189. https://doi.org/10.1007/s12161-018-1298-7

Rojas-Hernández, A., \& Ramírez-Silva, M. (2012). La enseñanza experimental de la Química General y la Química Analítica desde México (la Enep-Cuautitlán de la Unam y la UamIztapalapa) en el último cuarto del siglo xx. Educación química, 23, 136-140

Sevian, H., \& Fulmer, G. (2012). Student Outcomes from Innovations in Undergraduate Chemistry Laboratory Learning. Educación Química, 23, 149-161.

Sudhoff, M., Prinza, C., \& Kuhlenkötter, B. (2020). A Systematic Analysis of Learning Factories in Germany - Concepts, Production Processes, Didactics. Procedia Manufacturing, 45, 114-120. https://doi.org/10.1016/j.promfg.2020.04.081

Supasorn, S. (2012). Enhancing undergraduates' conceptual understanding of organic acid-base-neutral extraction using inquiry-based experiments. Procedia - Social and Behavioral Sciences, 46, 4643-4650. 
Yang, L., Said, R., \& Abdel-Rehim, M. (2017). Sorbent, device, matrix and application in microextraction by packed sorbent (MEPS): a review. Journal of Chromatography B, 1043, 33-43. https://doi.org/10.1016/j.jchromb.2016.10.044 